\title{
Teaching Spatial Science Courses in Public Universities in Tanzania: Challenges and Opportunities
}

\author{
Emiliana John Mwita \\ Geography Department, Dar es Salaam University College of Education, Dar es Salaam, Tanzania \\ Email: emi@duce.ac.tz,emmyrh@yahoo.com
}

Received September 23, 2013; revised October 23, 2013; accepted October 30, 2013

Copyright (c) 2013 Emiliana John Mwita. This is an open access article distributed under the Creative Commons Attribution License, which permits unrestricted use, distribution, and reproduction in any medium, provided the original work is properly cited.

\begin{abstract}
Spatial science courses that are Remote Sensing (RS) and Geographic Information System (GIS) are increasingly growing into extremely important disciplines that accommodate various applications in wider sectors of development. Effective teaching and learning of the courses, however, requires intensive investment in facilities and human resources, since the technology is sophisticated and growing fast. This study aims at exploring the challenges of teaching and learning spatial science courses, RS and GIS, particularly in public universities in Tanzania. The study also identifies possible opportunities to improve the situation. Using social survey techniques in data gathering and analysis and author's own experience, lack of resources, poor background to the courses, delivery methods, limited number of staff and large classes were seen to be the major obstacles in successful learning and teaching. Opportunities exist in using open source resources, collaboration with other institutions within and outside the country and for the universities to give due weight to the courses by building capacity of their staff and procuring facilities, especially laboratories equipments.
\end{abstract}

Keywords: GIS; Remote Sensing; Learning; Obstacles

\section{Background}

Remote Sensing (RS) and Geographic Information Systems (GIS) technology has evolved over the past few decades to assist professionals in many disciplines. This technology originated in land based applications but is now widely utilized in several diverse disciplines. Rapid development of computer hardware and software and sophisticated tools for capturing spatial information has accelerated the prominence of RS and GIS [1]. Education and teaching has played a vital role in RS and GIS transformation. Today RS and GIS technology is one of the important University academic programs offered in a variety of disciplines such as urban and transportation planning, agriculture, forestry, public health, social and environmental sciences. Modern software tools have assisted users in integrating the scientific information derived from RS into their own disciplines, in their own ways.

Application of RS and GIS is an integral aspect of planning, monitoring and management of environmental resources and other development activities [2]. Both RS and GIS are very strong tools in planning and decision making. They allow engineers to continuously monitor any change in any intended plans to secure their success or rectification to meet the requirements [3]. The strong power of the RS lies in its ability to gather a wide range of data inaccessible areas. RS also supplies the needed geo database to build informative and rich GIS. The importance of GIS is based on its role in storing and managing a lot of data about images and all related attributes to allow their manipulation, analysis and finally presentation according to choice. GIS has also great ability in presentation and facilitates the analysis, computations, prediction and retrieval through many types of processing, especially overlaying of different layers extracted from multi date remotely sensed data. The endless abilities of combining RS and GIS allow doing the most tedious work in a flexible, fast and accurate manner, especially with the availability of more and more satellite images and the improvement of their quality with time.

Worldwide, RS and GIS have been taught at various levels. In the United Kingdom, for example, RS and GIS courses vary at different levels such as undergraduate, postgraduate, and other special training programs [4]. Remote sensing technology is developing quickly, as shown by the occurrence of new sensors, new information sources, new applications and new information proc- 
essing methods. Special remote sensing career training programs are popular in many countries, including thematic tutorials, summer schools and short training courses. In China, for instance, RS education has been included in various programs such as Surveying, Mapping, Geography, GIS, Geology and Engineering. RS usually comprises one or two modules for these programs, in which both fundamental principles and practical applications are taught.

In Germany, RS is offered from secondary schools' level where all basics and fields of application of RS are taught [5]. Rwanda has introduced GIS studies in secondary schools since 2008 [6]. According to [7], GIS technology came into the public mainstream, and it was first taught in universities in the mid 1980s; indeed, the very first university level textbook on the subject was published in 1986. In Tanzania, RS and GIS are taught at the university level, although currently, some courses are offered at lower levels. In many public universities like the University of Dar es Salaam, RS has been taught for more than two decades either as modules in some courses or as standalone introductory courses, particularly in undergraduate programmes.

According to [1], effective teaching and learning of RS and GIS is required to develop skills at three different levels 1) a general awareness of the potential of tools for handling spatial data and their benefits; 2) an understanding of the conceptual basis of RS and GIs operations and analysis; and 3) technical skills with specific GIS/RS applications. However, there are several key barriers existing to the rapid attainment of these objectives. The first is the technical complexity of GIS principles and their sophisticated implementation in a computing environment. This factor has inhibited self-teaching and take-up of courses by those with traditional "spatial skills" such as cartographers, surveyors and geographers. The second barrier has been the small existing skills base for GIS, especially for those with skills in all three areas listed above. A number of hands on activities are also required for RS/GIS learners to be conversant with the programs and the best ways to handle the data. Effective teaching of these courses is also labour and resource intensive in terms of time and money. Large initial investment is required in computer and software installment. Efficient and reliable laboratories furnished with powerful computer hardware and software that are not cheap for data analyses are also required.

A number of challenges exist, which hinder effective teaching of RS and GIS courses. Some of problems are educational or institutional in character [5], others relate to the difficulty of providing adequate resources of computer hardware and software to support the teaching, and yet others relate to syllabus and curriculum design, knowing what to teach, how to teach it and in what order at what level. Geographers who have been working in higher education on RS and GIS will probably already have a clear idea of what might be involved but the newcomers to the field within geography or, more importantly teachers and students whose background does not include most of the traditional geographical components of GIS such as computer mapping, satellite remote sensing and analytical cartography may not have the same experience. The current study had to be undertaken to explore the challenges that both students and teachers are facing in learning and teaching the RS and GIS courses, and they are also facing available opportunities that can be used to overcome the challenges.

\section{Methodology}

This study involved lecturers and students of various public universities such as the University of Dar es Salaam (UDSM), Dar es Salaam University College of Education (DUCE) and Mkwawa University College of Education (MUCE), ARDHI University and University of Dodoma. The UDSM and ARDHI have a long experience in offering particular courses while DUCE, MUCE and UDOM are newly established universities (less than 10 years old) and thus, the assumption was that their experience may be different from that of the former. Other interested stakeholders who have been working in the field for longtime both from within and outside the country were also consulted.

In total, 155 respondents were consulted; these were purposively selected based on their background. Some 100 questionnaires were distributed to students to gather information on their experience in learning the courses, particularly with regard to the difficulties they were facing and the usefulness of the courses in their future career. Interviews (phone and face to face) were done but also relevant literature on teaching and learning RS and GIS were reviewed together with other reports, including students' course evaluation. The collected data were coded and entered into the computer for further analysis. The Statistical Package for Social Sciences (SPSS) 14.0 was used for analysis. Since the study was descriptive in nature, descriptive statistics such as frequency distributions and percentages were used for description of the data obtained from the participants in the field. Qualitative data were subjected to thematic or content analysis.

\section{Results and Discussion}

A number of challenges were identified by both students and lecturers that hinder effective learning and teaching of RS and GIS courses. Even though students had some specific factors different from their lecturers, $75 \%$ of the identified factors were related. The challenges identified were as follows: 
Fifty percent of the respondents indicated that the courses are taught more theoretically without involving some practicals. Students feel that the courses require practical rather than theory alone. Without enough practice, it is very difficult for students to understand the subjects adequately. Both students and lecturers argued that, RS and GIS are natural science related subjects, thus they require hand on tutorials for better comprehension. Lack of practical is a great obstacle in performance and application of the knowledge acquired. Lack of practical goes hand in hand with lack of teaching facilities. Many public institutions do not have laboratories equipped with computer hardware and software and simple equipment like global positioning systems as well as data sets like satellite imageries that are essential for learning the two courses. Twenty five percent of the respondents argued that RS and GIS laboratories are critically important in facilitating the teaching and learning process. The laboratories require much financial resource for smooth running that are largely unavailable due to meager budgets allocated to the universities by the government. Furthermore, where the laboratory exists, maintenance becomes a problem also due to shortage of money. One of the respondents had the following concerns:

"You know Geography is like any other natural science course, the problem is that many people categorise Geography as a social science, which makes it difficult to gain its weight. In such a case, there is no consideration for construction of laboratories like in other courses such as Biology, Chemistry or Physics. This is a great set back in successful teaching and learning of the subject".

[8] observed the importance of RS and GIS in increasing efficiency in planning and management in many aspects of development. They argue that enough financial resource is required to be invested for the successful use of spatial data and technology in general. In many instances, this has been too much for most of the developing countries. Lack of financial resources is an obstacle to invest in the technology and smooth running of the facilities required. The software required are also very expensive; for instance, ArcGIS software is sold at $10,000 \$$ with limited number of license, which may not serve our larger classes at once. [3] also see financial resources a stumbling block in the development of spatial sciences and further add to the difficulties of maintaining the laboratories, keeping its hardware running in a climate of ubiquitous dust, power fluctuations and other hazard that destroys both existing database and the hardware.

[9] identify poor background to GIS and technical aspect of the subject to be some of the problems in learning and teaching the subject that requires an improved curriculum to handle. Rwanda introduced GIS in secondary schools in 2007 and one of the major aims was to raise the level of awareness when students enter higher education [6]. In the current study, all respondents pointed out that they did not have a good background to the subject. Many also lack basic computer knowledge, which is essential for application of RS/GIS. Unlike many other subjects where students had previous experience in lower levels of education i.e. secondary or high schools, many students taking the two courses do not have appropriate background because most of them studied arts subjects. Even though they did Geography as one of their major, there were no specific modules to introduce them to these particular courses. This was also the concern of the lecturers, as most indicated that lack of background to the course is a great obstacle to the best performance in the subject. The lecturers also said they see a significant difference between students with Physics background and those without. This is because RS, for example, draws its background from Physics and Mathematics as well. One of the respondents had this to say;

"I don't have any background to the courses; these courses require some good background in Physics and Mathematics as I can see modeling elements. I left Mathematics in Form Four and Physics in Form Two, thus I have to use extra effort to pass the course".

Fifteen percent of the respondents also complained about the way the course is delivered. The common teaching method used most often is lecturing; the lectures are given in a very fast speed which makes it difficult for learners to follow. Many of the terminologies used are also not very familiar. Furthermore, time allocated for the courses is also sometimes not enough. Lack of field work experiences and use of at least images or teaching aids to teach makes the course much harder.

Even though students may complain about lecturing method, it may still be an important technique for initial understanding of the concepts. [5] think interactive learning is most important for efficient learning and teaching. In addition, the use of satellite images in class offers more practical experience, in addition to strengthening natural science education. Other advantages include high degree of descriptiveness, motivating effect of the imagery ways for problem-oriented working, the strengthening of spatial orientation competence, method competence and evaluating and practice skills. Another factor which accounted for the rest 5\% was negative attitude towards the course. It is also not our culture to think spatially. Indeed, much of the analysis done in many aspects undermines location, thus it is difficult for some students to cope with the course. There are also some students with negative perception on the course due to information overheard from previous students or their perception towards science related subjects. Many students also lack experience in using computers, which means even when they are given hands on activities, it is not easy for them 
to carry on the exercises. In addition, some of the students do not see the importance of the courses in their lives and career in general.

Many universities in Tanzania, particularly public universities have been aiming at increasing enrolment but the human resources and facilities available are not in a position to accommodate the increased number of students. According to the lecturers, the number of students enrolled in the course is large compared to the staff available and time allocated for teaching the course. In UDOM, for instance, the number of students ranged between 120 and 430 in the academic year 2012/2013, UDSM had more than 1000 and MUCE and DUCE had between 250 and 350 respectively. In most cases at UDSM, one lecturer is involved in teaching with at most two tutorial assistants to run seminars. These classes are too large for such an involving course, thus there is a need to improve the facilities or minimize the number of students enrolled in the course. Some lecturers had the following concerns:

"I have hard time teaching the course without practicals, students also have difficult time learning but sometimes I think even if I had opportunity to run practicals, with more than 1000 students and the limited number of staff available, how could I have managed to handle the class? We have to look for alternative solution, if we want to improve knowledge and performance of our students".

[10] contend that spatial data is scanty and that most geospatial data sets are still in analogue form, are not regularly updated, and their consistency across organizations still needs to be verified. Internet connections available are also not strong enough to support access to free data sets like Landsat. At times, it may take up to four or more hours to download a single image and, in most cases; one may need to download data sets at night, when not many people are using the service. This situation affects the lecturers a lot and consumes much of their time that they would otherwise invest in intensive preparation of their lessons. There is also too much bureaucracy in the procurement of laboratory facilities and maintaining the laboratories where they exist.

Despite the challenges, many of the respondents still think there are possibilities for improvement in teaching and learning environment. Lecturers need to be more creative and motivate students by trying to use better teaching methodologies than lecturing alone. Opportunities are there to take students for tour to areas where RS or GIS is applied in order for them to learn more and get a real life experience. Visiting such places as meteorological stations and surveying and mapping departments may help students improve their understanding. Use of videos and power point presentation for larger classes can allow students to learn more easily. Where possible, hard copy images should be used for teaching to increase students' interpretation and analytical skills.

Today, there are so many free softwares that can be downloaded and installed as standalone in personal computers e.g. GRASS and quantum GIS. Where computers are available, it is very possible for lecturers to run their tutorials without much difficulty. A number of students in higher learning institutions also have their private laptops where they can install these programs so that they make use of them even in their own private time. Many tutorials are also available online together with much free literature that students can use to improve their knowledge on the subject. Students should be encouraged to visit such websites like NASA, ESRI and ERDAS where they can access tutorials that are well elaborated with animations for their better understanding.

Those responsible for the most successful implementations of GIS in any area know that adequately-trained personnel are a fundamental requirement [3]. Training specialists know that computer technology, particularly technology as sophisticated as GIS, may need considerable training time for people in less material/technological cultures than in countries that are exporting this technology. There are more opportunities to train for short and long courses within and outside the country. Some of the courses are offered for free, for instance, ERDAS software suppliers in East Africa have been offering free courses to RS \& GIS specialists. Lecturers specializing in these subjects may make use of such opportunities to keep update with the frequent changing technologies.

Investment in data processing and metadata building to make data searchable and sharable is crucial for effective teaching and learning. Opportunities are also available to share data and other resources between institutions, if they build partnerships with both internal and foreign universities. ESRI, for instance, have been offering student version ArcGIS to many universities in developed countries. Efforts could be made to link up with companies producing soft and hardware for spatial sciences and seek their support in improving teaching facilities.

\section{Conclusion and Recommendation}

This paper presented the challenges and opportunities of teaching spatial science courses i.e. RS and GIS in public universities in Tanzania. Learners, lecturers and many other interested stakeholders were involved in the study. The major challenges identified were lack of teaching and learning facilities, poor background to the courses, poor methods of course instruction, increased enrolment with limited number of staff and poor access to available free resources. There are, however, several opportunities to improve the prevailing situation by introducing the 
courses in the lower learning levels like secondary schools, using e-learning facilities and teaching methods that accommodate large classes and investment in open source software and open data forums. Others include making use of internet sources and initiating collaboration with spatial science related institutions, both within and outside the country. It is recommended that the universities set aside enough financial resources to support installation of laboratories and other teaching facilities as well as human resource capacity building. This will not only improve performance but also generate income through provision of tailor made courses to many other people who are interested in the course. It is also important to introduce spatial science courses in lower levels of education including secondary schools to improve their understanding and increase their interest in the course at advanced levels.

\section{REFERENCES}

[1] J. Raper and N. Green, "Teaching the Principles of GIS: Lessons from the GIS Tutor Project," International Journal of Geographical Information Systems, Vol. 6, No. 4, 1992, pp. 279-290.

[2] K. White, "Book Reviews: Asrar, G., Editor, 1989: Theory and Applications of Optical Remote Sensing. Chichester: John Wiley \& Sons Ltd. xiv + 740 pp. 58.50 Cloth," Progress in Physical Geography, Vol. 14, No. 4, 1990, pp. 559-560.

[3] D. A. Hastings and D. M. Clark, "GIS in Africa: Problems, Challenges and Opportunities for Co-Operation," International Journal of Geographical Information Systems, Vol. 5, No. 1, 1991, pp. 29-39.
[4] P. Du, P. Aplin and M. Jackson, "Remote Sensing Education in China and the UK: Synergy and Opportunities," The International Archives of the Photogrammetry, Remote Sensing and Spatial Information Sciences, Vol. 37, Part B6a, 2008.

[5] K. Voss, R. Goetzke and H. Hodam, "Learning Modules-A Way to Integrate Remote Sensing Methods in School Education," The International Archives of the Photogrammetry, Remote Sensing and Spatial Information Sciences, Vol. 34, No. 30, 2008, p. 5.

[6] M. Forster and T. Mutsindashyaka, "Experience from Rwandan Secondary Schools Learning GIS,” Presented at the ESRI Education User Conference, International GIS Education Experience, 2008, p. 6.

[7] G. C. Mulaku, "Professionalism in GIS: International Aspects of the Certification Debate," Journal of Geographic Information System, Vol. 5, No. 4, 2013, pp. 347-353. http://dx.doi.org/10.4236/jgis.2013.54033

[8] L. Kithsir Perera and R. Tateishi, "Do Remote Sensing and GIS Have a Practical Applicability in Developing Countries? (Including Some Sri Lankan Experiences)," Remote Sensing, Vol. 16, No. 1, 1995, pp. 35-51. http://dx.doi.org/10.1080/01431169508954370

[9] D. J. Unwin and Eight Others, "A Syllabus for Teaching Geographical Information Systems,” International Journal of Geographical Information Systems, Vol. 4, No. 4, 1990, pp. 457-465.

[10] G. C. Mulaku, J. B. K. Kiema and D. N. Siriba, "Assessment of Kenya's Readiness for Geospatial Data Infrastructure Take Off,” Survey Review, Vol. 39, No. 306, 2007, pp. 328-337.

http://dx.doi.org/10.1179/175227007X197237 